\title{
Androgènes et sexualité masculine
}

\author{
A. HAFIDI, M.H. GHARBI
}

Service d'Endocrinologie et Maladies Métaboliques, C.H.U. Ibn Sina, Rabat, Maroc

\section{RESUME}

La testostérone ( $T$ ) est indispensable au maintien de la fonction sexuelle chez l'homme. Elle agit sur toutes les composantes de l'acte sexuel: libido, érection et éjaculation. Au niveau de l'érection, seules les érections spontanées nocturnes sont androgénodépendantes à l'inverse de celles provoquées par fantasmes ou par stimulation visuelle.

C'est essentiellement sous forme de dihydrotestostérone (DHT) que l'hormone mâle semble exercer ses actions aussi bien au niveau central que périphérique. Une intervention au niveau cérébral des estrogènes $(E)$ produits localement ne peut cependant pas être formellement écartée.

Les concentrations de $T$ requises pour le rétablissement de la fonction sexuelle en cas d'hypogonadisme sont proches de la limite inférieure des valeurs normales. le seuil supérieur d'action de la $T$ sur la sexualité - s'il existe- se situerait à des niveaux supraphysiologiques.

L'androgénothérapie des dysfonctionnements sexuels est en pleine évolution. De nouveaux produits plus performants et de nouvelles voies d'administration moins astreignantes et plus physiologiques sont en cours d'évalua- tion. De plus l'affinement de l'exploration des androgènes plasmatiques associé à une meilleure compréhension des effets secondaires de la thérapeutique androgénique laissent espérer l'obtention dans un proche avenir d'un consensus autour de l'élargissement du champ d'indications de l'androgénothérapie.

Mots clés : Androgènes, intérêt sexuel, érection, éjaculation, prostate, risque cardiovasculaire.

Si le rôle joué par les testicules dans la sexualité masculine est bien connu à travers les effets de la castration pratiquée depuis l'antiquité, l'intervention de la $\mathrm{T}$ au niveau des différentes composantes de cette sexualité demeure encore imparfaitement élucidée. Néanmoins, sur le plan pratique, les androgènes sont largement, voire abusivement, utilisés dans le traitement des troubles sexuels. Comme l'androgénothérapie n'est pas complètement dénuée de risques, il devient impératif de tenter d'en préciser les modalités et les indications à la lueur des dernières acquisitions physiopathologiques et pharmacologiques.

\section{ASPECTS PHYSIOPATHOLOGIQUES}

1. Rôle de la testostérone dans le maintien de l'activité sexuelle (2)

Les expériences de castration effectuées chez l'animal ont clairement démontré - 
depuis déjà plusieurs décennies- l'importance des perturbations sexuelles qui en résultent et qui peuvent aller jusqu'à l'extinction totale de toute activité sexuelle. Elles ont notamment mis en évidence une dissociation entre l'évolution de l'imprégnation androgénique - jugée sur la testostéronémie - et celle des désordres sexuels. Ces derniers s'installent en effet de façon progressive et différée par rapport à la chute rapide de la $\mathrm{T}$ plasmatique consécutive à la castration. Ainsi l'extinction complète de la fonction sexuelle après castration demande un délai de quelques semaines à plusieurs années. Ce délai varie plus en fonction de l'espèce considérée et du degré d'expérience sexuelle individuelle au sein d'une même espèce, que de l'imprégnation androgénique. Toute suppléance par les androgènes surrénaliens étant exclue sur la base des données d'expériences de surrénalectomie associée à la castration.

Chez l'homme jeune, les résultats d'expériences de castration biochimique transitoire par des analogues du GnRH [16] se révèlent conformes à ceux de l'expérimentation animale. En effet la T s'effondre dès les premiers jours suivant la castration, tandis que les premières manifestations de dysfonctionnement sexuel n'apparaissent qu'à partir de la 2ème semaine. Il s'agit d'abord d'une diminution de la libido et des fantasmes occasionnant une baisse de la fréquence des rapports sexuels. Plus tard, entre la 4 ème et la 6ème semaine, la fréquence des masturbations et des érections spontanées diminue. Toutefois la capacité de maintien des érections obtenues après rapport sexuel ou après masturbation reste conservée.

La réversibilité de l'ensemble de ces perturbations après trois semaines d'androgénothérapie atteste que la $\mathrm{T}$ est indispensable au maintien d'une activité sexuelle normale chez l'homme.

\section{Effets de la testostérone sur les dif- férentes étapes de l'acte sexuel}

\section{a) Au niveau de la libido}

L'influence de l'imprégnation androgénique sur le niveau de l'intérêt sexuel est largement démontrée $[2,13,16,23]$. Les états de privation androgénique s'accompagnent d'un déclin de la libido dont la restauration est assurée par la substitution androgénique. Cette action de la $\mathrm{T}$ s'exercerait chez le rat principalement au niveau du noyau préoptique médian et de l'hypothalamus antérieur à travers une modulation essentiellement dopaminergique [44]. Une revue récente des effets de la dopamine sur le comportement sexuel est en faveur d'un rôle facilitateur mais qui s'exercerait plus sur la composante érectile que sur l'intérêt sexuel [14].

\section{b) Au niveau des érections}

L'action de la $\mathrm{T}$ sur l'érection est encore objet de discussion. Deux hypothèses s'affrontent :

- La première dénie à la $\mathrm{T}$ toute influence directe sur le phénomène érectile; le déclin de l'érection dans les situations de privation androgénique étant considéré comme une conséquence de la baisse du désir sexuel. Cette hypothèse se base sur les arguments suivants :

- La similitude des réponses érectiles aux fantasmes sexuels et aux films érotiques chez les hommes hypogonades, qu'ils soient ou non substitués en androgènes [27].

- L'enregistrement, après stimulation visuelle, de réponses érectiles semblables, aussi bien en terme de tumescence que de rigidité, chez les hommes hypogonades non substitués et les sujets normaux [15].

- La conservation de la capacité de maintien des érections dans les situations d'hypogonadisme masculin expérimental [16]. 
- La deuxième hypothèse plaide au contraire en faveur d'un impact direct de la $\mathrm{T}$ au niveau des mécanismes de l'érection. Elle s'appuie sur des arguments biochimiques, anatomiques et cliniques :

- Mise en évidence de récepteurs aux androgènes au niveau des neurones du noyau parasympathique sacré et au niveau des terminaisons médullaires des afférences péniennes [23].

- Effet trophique de la testostérone sur la croissance du soma et des dendrites des neurones des centres érectiles de la moelle sacrée [23].

- Détection de profondes altérations des érections spontanées nocturnes sous forme d'une baisse importante de la rigidité et de la tumescence chez les hypogonades non substitués par rapport aux sujets normaux. Ces perturbations régressent après substitution androgénique [15].

En fait ces deux hypothèses s'avèrent plutôt complémentaires dans la mesure où elles concernent deux types distincts d'érections obéissant chacun à une régulation différente. On admet actuellement le caractère androgénodépendant des érections spontanées nocturnes et androgéno-indépendant des érections obtenues après stimulation visuelle.

\section{c) Au niveau de l'éjaculation}

L'expérimentation sur différentes espèces animales a montré que les premières perturbations sexuelles engendrées par la castration intéressent l'éjaculation qui reste la dernière à se corriger après administration d'androgènes [2]. Chez l'homme hypogonade l'arrêt du traitement substitutif est suivi d'une diminution du volume de l'éjaculat puis d'un retard de l'éjaculation qui finit par disparaître [13].

On peut donc affirmer, contrairement à ce qui est actuellement admis, que l'action de la $\mathrm{T}$ sur l'activité sexuelle ne se limite pas à l'excitation sexuelle, mais s'exerce également au niveau de l'érection et de l'éjaculation.

\section{Formes actives et seuils de la testo- stérone}

Si le rôle de la $\mathrm{T}$ sur la fonction sexuelle est actuellement mieux analysé, des questions persistent sur sa forme active et sur les concentrations plasmatiques nécessaires au rétablissement ou à l'amélioration de la fonction sexuelle.

Chez l'homme comme chez la plupart des primates la DHT semble la forme active aussi bien au niveau central que périphérique [13]. Les résultats d'études préliminaires sur l'utilisation de la $5 \alpha$-réductase $(5 \alpha-R)$ chez l'homme tendent à jeter le doute sur cette hypothèse mais ils font encore l'objet de nombreuses réserves [42]. Le rôle prépondérant de l'estradiol (E2) au niveau central suggéré par son efficacité à rétablir le comportement sexuel chez le rat et par la présence d'une aromatase cérébrale, est actuellement remis en question. En effet l'utilisation de tamoxifêne (anti-estrogène) ou de testolactone (inhibiteur de l'aromatase), qui créent une déplétion sélective en estrogènes avec respect des niveaux de $T$, n'affecte nullement le comportement sexuel de l'homme normal ou castré et supplémenté en T [16, 19]. Il faut cependant souligner que certains effets des stéroïdes gonadiques sur le comportement sexuel résultent de leur action sur le système nerveux central et qu'il persiste des incertitudes quand à la perméabilité de la barrière hématoméningée à la testolactone. Par conséquent une éventuelle intervention des estrogènes produits localement au niveau du SNC ne peut être exclue. D'ailleurs d'après Luisi et Franchi [30] l'utilisation par voie orale d'un androgène aromatisable (undécanoate de $\mathrm{T}$ ) semble plus efficace que celle d'un androgène non aromatisable (mestérolone) sur la restauration de la libido et des érections chez les hommes hypo- 
gonades. D'un autre coté il a été rapporté la persistance d'éréctions matinales et de "pollutions" nocturnes chez un patient présentant une résistance aux estrogènes par mutation du gène de leur récepteur nucléaire classique [45]. Or il a été récemment démontré que les stéroïdes peuvent également agir par un mécanisme non génomique à travers une activation des membranes cellulaires [20]. Par conséquent la seule certitude qui persiste et qui est relative à l'action des estrogènes est l'absence de leur effet au niveau périphérique.

D'autre part le degré d'imprégnation androgénique requis pour le rétablissement ou l'optimalisation des performances sexuelles reste également discuté. Les expériences effectuées chez l'animal [23] ont établi deux seuils d'activité de la T ; un seuil inférieur en deçà duquel toute activité sexuelle est abolie et un seuil supérieur au delà duquel aucune amélioration des performances sexuelles ne peut être obtenue. Ce dernier correspond à des concentrations supraphysiologiques de T. Chez l'homme, il est établi, qu'une androgénothérapie qui assure des concentrations plasmatiques relativement faibles de $\mathbf{T}$ (proches de la limite inférieure des valeurs normales) suffit à restaurer l'activité sexuelle en cas d'hypogonadisme. Il n'en est pas de même pour l'amélioration des performances sexuelles par utilisation d'androgènes chez l'homme eugonadique. Les premiers essais étaient en faveur de l'existence d'un seuil supérieur de la testostéronémie se situant entre 2 et $4.5 \mathrm{ng} / \mathrm{ml}$ au delà duquel toute augmentation des doses est inopérante $[4,42]$. Les résultats d'études plus récentes sont contradictoires. Les unes montrent que des doses pharmacologiques de $\mathrm{T}$ sont encore capables d'améliorer ou d'augmenter significativement le comportement sexuel de patients eugonadiques atteints ou non de dysfonctionnement sexuel $[5,6,34,37]$. D'autres n'enregistrent qu'un effet discret de stimulation psychosexuelle sans augmentation de l'activité sexuelle lors de l'uti- lisation de doses supraphysiologiques de T chez des hommes normaux $[1,17]$.

\section{ASPECTS THERAPEUTIQUES}

\section{Produits}

Les produits disponibles dans le cadre de l'androgénothérapie sont presqu'exclusivement représentés par les esters de la T. Ceux-ci sont administrés par voie orale ou parentérale (intramusculaire) et plus récemment transdermique, ou sous forme d'implants sous cutanés ou intradermiques.

La voie orale a déçu car elle nécessite l'utilisation de produits alkylés dont la toxicité a entraîné l'abandon. Par ailleurs les nouveaux produits tel que l'undécanoate de $T$, certes beaucoup mieux tolérés, présentent le double inconvénient de la prise pluriquotidienne et de l'irrégularité de l'absorption digestive [40].

La voie parentérale demeure la plus utilisée, compte tenu des limites de la voie orale, de l'impopularité des implants et du manque de recul pour la voie transdermique. Cependant elle souffre de son incapacité à assurer des concentrations plasmatiques physiologiques et stables de T. En effet des mesures répétées de $T$ sériques, corroborées par une simulation pharmacocinétique assistée par ordinateur [36], ont clairement démontré que les différentes substitutions communément utilisées donnent des taux initiaux supraphysiologiques suivis de taux subnormaux au moment de l'injection suivante. Ces oscillations sont mal vécues par les patients au niveau de leur sexualité et les amènent à augmenter les doses et/ou la fréquence des injections. Ce problème pourrait être résolu si des essais thérapeutiques plus nombreux confirment les données préliminaires sur les propriétés du dernier-né des esters de T.

Le buciclate de $T$ semble en effet posséder des caractéristiques pharmacocinétiques très favorables qui lui permettent d'assu- 
rer, à la dose de $600 \mathrm{mg} / \mathrm{semaine,} \mathrm{des}$ concentrations plasmatiques de $\mathrm{T}$ physiologiques et stables [8]. D'autre part, les essais initiaux d'utilisation IM de T sous forme de microsphères biodégradables ont donné des résultats intéressants chez les primates non humains. Les essais chez l'homme sont en cours $[9,12]$.

La voie transdermique par application scrotale quotidienne de patchs contenant $10 \mathrm{ou}$ $15 \mathrm{mg}$ de $\mathrm{T}$ permet également d'obtenir un profil plasmatique satisfaisant $[3,7]$. Elle semble de plus, très bien acceptée par les patients qui la préfèrent aux injections. Cependant elle modifie la métabolisation de la $T$ et aboutit dans certains cas à des taux supraphysiologiques de DHT [40]. D'autres études sont donc nécessaires avant de conclure à son innocuité.

Les implants intradermiques de $\mathrm{T}$ ont une pharmacocinétique favorable [22]. Néanmoins leur insertion requiert une intervention chirurgicale mineure et ils peuvent être occasionnellement exclus. Ils n'ont donc pas connu une large diffusion en pratique.

\section{Risques potentiels}

\section{a) Au niveau de la prostate}

De lourds soupçons subsistent quant à la participation de l'androgénothérapie dans la genèse de la pathologie tumorale prostatique. Ils se fondent sur le rôle physiologique joué par les androgènes dans la croissance normale de la prostate. Néanmoins les preuves objectives de leur intervention au niveau de ces processus pathologiques demeurent insuffisantes [7].

\section{- hypertrophie bénigne de la prostate (HBP)}

Les résultats favorables des tentatives de traitement de l'HBP par les antiandrogènes, les analogues du GnRH ou les inhibiteurs de l'activité $5 \alpha$-réductasique constituent une preuve en faveur de l'implication des androgènes dans la genèse de cette pathologie [7]. Cependant la relation de l'HBP avec les androgènes parait à priori paradoxale puisqu'on assiste à une croissance exagérée d'un organe androgénodépendant alors que le signal androgénique reste inchangé ou même diminue chez le sujet âgé. Ce paradoxe peut être levé si l'on admet que la sensibilité aux androgènes augmente progressivement avec l'âge. Deux arguments appuient cette hypothèse :

- l'importance de l'activité $5 \alpha$-réductasique au niveau des fibroblastes de prostates adénomateuses par rapport aux contrôles [11, 35]

- la diminution du volume de ces mêmes prostates traitées par les inhibiteurs de la $5 \alpha$ réductase [33].

Néanmoins des travaux plus récents rapportent une baisse de la sensibilité du tissu adénomateux aux androgènes, avec une diminution de l'ARN messager de leur récepteur et de la $5 \alpha$ réductase de type I et II [10]. Ces contradictions apparentes peuvent s'expliquer par une grande variabilité de l'activité $5 \alpha$-réductasique prostatique en fonction de la zone considérée [11]. Par ailleurs l'augmentation des facteurs de croissance avec l'âge pourrait constituer, à l'instar de l'augmentation de l'activité $5 \alpha$ réductasique, un facteur d'amplification de la sensibilité aux androgènes. Cependant leur taux, bien que s'élevant avec l'âge, reste similaire dans le tissu normal et adénomateux [10]. D'autres études s'avèrent donc nécessaires pour lever ce paradoxe. Par ailleurs il a été suggéré, sur la base de données expérimentales, une implication des estrogènes $(\mathrm{E})$ dans la genèse de l'HBP. Chez l'homme de nombreuses constatations appuient cette thèse : les taux circulants d'E augmentent avec l'âge et cette augmentation est plus importante en cas d'HBP. De plus leurs niveaux ont été trouvés plus élevés dans le tissu adénomateux que dans le tissu prostatique normal et leur fixation se fait électivement dans les noyaux du stroma qui est reconnu comme le point de 
départ de l'HBP. Néanmoins la concentration des récepteurs $(\mathrm{Rc})$ aux $\mathrm{E}$ de haute affinité a été trouvée basse dans l'HBP humaine contrairement à ce qui se passe dans le modèle animal (chien). -Elle pourrait cependant suffire à l'exercice de leur activité biologique. L'hypothèse de l'intervention des $\mathrm{E}$ (provenant de l'aromatisation des androgènes) dans la genèse des adénomes de la prostate a débouché sur l'utilisation des inhibiteurs de l'aromatase dans le traitement de l'HBP [32].

\section{- Cancer de la prostate (Kc P)}

C'est la tumeur maligne la plus fréquente chez l'homme. La prévalence des micro-carcinomes augmente avec l'âge et atteint 50\% à la septième décade de la vie.

Des études faites chez l'animal semblent en faveur d'un rôle promoteur des androgènes sur le $\mathrm{Kc} \mathrm{P}$ au stade infra-clinique [7].

D'autres plus récentes effectuées sur des lignées cellulaires issues de métastases ganglionnaires(LNCaP) et osseuses (ALVA101) de $\mathrm{KcP}$ androgénodépendant permettent de mieux appréhender le mécanisme d'action des androgènes dans ce processus. Cette action serait principalement médiée par des facteurs de croissance agissant par un effet autocrine ou paracrine. En effet il a été démontré que ces cellules possèdent des récepteurs à ces facteurs de croissance et sécrètent l'IGF1 et sa protéine de liaison I'IGFBP3 [41]. De plus il a été rapporté que l'utilisation d'anticorps monoclonaux dirigés contre les récepteurs EGF/TGFa bloque la prolifération des cellules (ALVA101) induite par la DHT [29].

L'action des androgènes sur ces facteurs de croissance s'exercerait à travers une modification des activités enzymatiques de la cellule cancéreuse aboutissant à une augmentation du rapport antigène spécifique prostatique (qui est une sérine protéase)/phosphatase acide prostatique (PSA/PAP). L'augmentation du PSA entraîne celle de la forme libre de l'IGF1 par clivage de
l'IGFBP3, tandis que la diminution de la PAP provoque une accumulation des phosphotyrosines : protéines clés de la prolifération cellulaire [41]. Ainsi les androgénes agissent en augmentant la forme libre des facteurs de croissance et en potentialisant leur effet sur la croissance des cellules cancéreuses. Par conséquent, puisqu'à l'heure actuelle il demeure très difficile d'écarter l'implication des androgènes -même à des concentrations physiologiques-dans la pathologie tumorale prostatique, il paraît prudent de recommander un monitorage de la croissance et de l'activité enzymatique prostatique lors de toute androgénothérapie au long cours. Il faut également souligner que le $\mathrm{KcP}$ est une contre-indication absolue à l'androgénothérapie au même titre que les autres cancers hormonodépendants de l'homme (sein et testicules).

\section{b) Au niveau du risque cardiovasculai- re [21]}

Il est bien établi que la mortalité cardiovasculaire est 5 fois plus importante chez l'homme que chez la femme en période d'activité génitale. Ceci a longtemps été mis sur le compte de l'action potentiellement athérogène des androgènes. Cette responsabilité de l'hormone mâle était confortée par les modifications lipidiques engendrées par l'utilisation des anabolisants et des androgènes administrés par voie orale. Des études récentes $[7,21]$ ont non seulement jeté le doute sur le caractère athérogène du profil lipidique masculin caractérisé par une augmentation des triglycérides(TG) et une baisse du HDL cholestérol(HDLCT) mais elles ont surtout écarté la responsabilité des androgènes endogènes dans la constitution de ce profil. En effet il a été rapporté, que contrairement à ce qui est constaté chez la femme, le sulfate de déhydroépiandrostérone(SDHEA), la testostérone totale(TT) et la testostérone libre(TL) sont corrélés significativement à une augmentation du HDLCT et à une diminution des TG chez l'homme. Le coefficient de cor- 
rélation entre androgènes et HDLCT est cependant trop faible $(0.2)$ pour proposer l'utilisation de la $\mathrm{T}$ dans la prévention du risque cardiovasculaire.

Dans ce sens, il est intéressant de signaler que, toujours par opposition à ce qui se passe chez la femme, chez qui l'obésité androïde s'accompagne d'une insulinorésistance et d'une baisse de la SHBG responsable d'une augmentation de la fraction libre de la $\mathrm{T}$, chez l'homme la corrélation entre anomalies de la distribution des graisses (dépôts au niveau de l'abdomen) et SHBG est faible $[38,39,46]$. Elle rend compte, du moins partiellement, de l'absence de relation voire même d'une relation inverse entre la testostérone et l'insuline [21]. Cette différence entre les sexes au niveau de l'insulinosécretion et de l'insulinorésistance est retrouvée chez l'animal. Le rat femelle traité par la $\mathrm{T}$ développe une insulinorésistance alors que celle-ci est provoquée chez le mâle par la castration et est améliorée par l'administration de faibles doses de $\mathrm{T}[24,25]$. A noter que les fortes doses n'entraînent pas d'aggravation de l'insulinorésistance du rat mâle castré. D'un autre coté, il faut souligner que l'obésité est responsable d'une diminution de la SHBG [47] au même titre que l'hyperinsulinisme qui diminue l'expression de son gène au niveau des hépatocytes [26]. Cette constatation doit être prise en compte dans l'interprétation des testostéronémies en cas de surcharge pondérale ou d'hyperinsulinisme.

Il ressort donc que l'action des androgènes sur les fractions lipidiques et l'insulinosécrétion chez l'homme -contrairement aux idées jusque là admises- ne favorise pas le processus d'athérogenèse et semble même s'opposer à plusieurs de ses facteurs de risque. Néanmoins il faut préciser qu'en ce qui concerne les androgènes exogènes l'effet sur le métabolisme lipidique varie selon le type de molécule, la voie d'administration et la dose choisis. Les anabolisants de syn- thèse administrés par voie orale ont un impact défavorable sur les fractions lipidiques plasmatiques (augmentation du LDL et diminution du HDLCT) alors que les esters de T, utilisés par voie IM et à dose physiologique respectent, voire même améliorent, le profil lipidique. Ceci serait en rapport d'une part, avec l'absence d'un passage hépatique premier en cas d'utilisation de la voie parentérale et d'autre part avec la conversion rapide des esters de $T$ injectables en estradiol ce qui n'est pas le cas des dérivés alkylés $[7,21]$. Toutefois les fortes doses d'esters de $\mathrm{T}$ sont pourvoyeuses d'un profil athérogène comme le démontre les résultats d'essais utilisant des doses supraphysiologiques d'esters de $\mathrm{T}$ dans le cadre de la contraception masculine [17].

Par ailleurs d'autres paramètres de pronostic cardio-vasculaires peuvent êtres influencés par les androgènes. L'apnée du sommeil est aggravée par l'administration d'androgènes même à doses physiologiques lors de substitution de situations d'hypogonadisme. Les androgènes peuvent être également responsables d'augmentation modérée de la masse sanguine qui n'atteint cependant la polycythémie franche qu'en cas d'association à une insuffisance respiratoire [7].

\section{c) Au niveau hépatique}

La T et ses esters ne sont pas hépatotoxiques contrairement aux dérivés de substitution en 17 alpha. Ces derniers peuvent être responsables de cholestase intrahépatique qui cède à l'arrêt du traitement. Ils ont également été incriminés dans la genèse de tumeurs hépatiques bénigne et maligne.

\section{d) Autres effets indésirables}

Une rétention hydrosodée peut être observée en cas d'utilisation de fortes posologies d'androgènes. Cet effet secondaire impose la prudence en cas d'utilisation des androgènes chez les insuffisants cardiaques, rénaux, chez les hypertendus et les migraineux. 
Par ailleurs les fortes doses de testostérone provoquent une diminution de la spermatogenèse par inhibition de la sécrétion des gonadotrophines. Cet effet est à la base de leur utilisation dans la contraception masculine.

\section{Indications}

\section{a) Dans l'hypogonadisme}

Le but du traitement substitutif est de corriger l'ensemble des troubles cliniques en rapport avec le déficit hormonal. En pratique cet objectif n'est atteint, avec les produits injectables actuellement disponibles, qu'au prix de concentrations supraphysiologiques de T. Des espoirs d'une androgénothérapie plus physiologiques sont annoncés avec les nouvelles voies d'administration (transdermique) et les nouveaux esters de T. La régression des troubles sexuels survient en général dès la troisième semaine de traitement.

\section{b) En cas d'eugonadisme}

Si la nécessité de la substitution androgénique en cas d'hypogonadisme relève de l'évidence, l'administration d'androgènes dans les situations d'eugonadisme fait encore l'objet d'une grande controverse. Elle soulève la question de la réalité de l'effet bénéfique de cette thérapeutique et met l'action sur ses effets néfastes potentiels notamment au niveau de la prostate.

Les travaux de ces dernières années sont comme signalé ci dessus- en faveur de l'absence d'un seuil supérieur de l'action de la $T$ au niveau de certains aspects du comportement sexuel, Ceci constitue donc une base à l'utilisation de l'androgénothérapie dans certains troubles du comportement sexuel même en situation d'eugonadisme définie par la normalité de la testostérone plasmatique. Il faut cependant garder à l'esprit que si les conséquences d'une surcharge androgénique peuvent être évitées au niveau cardiovasculaire par un choix judicieux des molécules et des voies d'administration elles demeurent imprévisibles au niveau prostatique. Pour cette raison une sélection rigoureuse des patients (bilan hormonal, recherche d'éventuelles contre-indications aux androgènes) doit être exigée assortie d'une surveillance régulière notamment prostatique.

Des études récentes $[13,18,28,31]$ montrent en effet que la $\mathrm{T}$ plasmatique n'est pas un paramètre suffisamment sensible de l'évaluation du statut androgénique. Celuici serait mieux approché par le dosage concomitant de la SHBG ou mieux par celui de la $\mathrm{T}$ biodisponible. Cette nouvelle approche permet de détecter des situations de déficits androgéniques caractérisées par une augmentation de la SHBG et/ou une baisse de la $\mathrm{T}$ biodisponible chez des personnes étiquetées comme eugonadiques sur la base de taux normaux de $\mathrm{T}$ totale. Ainsi dans le groupe des patients "eugonadiques" ( $T$ totale normale), souffrant de désordres sexuels, seront candidats au traitement androgénique, ceux qui ont une augmentation de la SHBG et ou une diminution de la testostérone biodisponible. De telles perturbations du statut androgénique semblent plus fréquentes parmi les personnes âgées $[18,28]$ et celles souffrant d'impuissance idiopathique quoique retrouvées de façon plus discrète dans la plupart des étiologies des dysfonctionnements sexuels (vasculaires, neurologiques, psychogènes) à l'exception de l'étiologie iatrogène [18].

Chez les sujets âgés l'androgénothérapie peut être potentiellement dangereuse étant donné le risque plus grand de survenue d'un cancer de la prostate. Pour cette raison l'indication $\mathrm{du}$ traitement doit prendre en compte les bénéfices escomptés en dehors de la sexualité (traitement de l'ostéoporose, correction des troubles du sommeil, de l'asthénie ...). Elle doit en outre être accompagnée d'un monitorage prostatique biochimique et échographique.

Chez les patients jeunes, la non disponibilité en routine des dosages spécialisés peut faire discuter l'opportunité de la mise en 
route d'un test thérapeutique de quelques mois sous réserve d'utilisation de doses physiologiques assortie des précautions d'usage en matière de surveillance. Les traitements au long cours ne sont à entreprendre que dans le cadre d'essais cliniques visant à apprécier les avantages et les inconvénients de cette thérapeutique afin de mieux en préciser les indications.

\section{CONCLUSION}

Une recherche très active dans le domaine de l'androgénothérapie et de la sexualité a permis :

- d'élaborer un profil particulier des désordres sexuels causés par la carence androgénique.

- de proposer de nouveaux produits et de nouvelles voies d'administration plus physiologiques.

- d'élargir le champ d'indication de l'androgénothérapie qui pourrait se justifier même en dehors de tout hypogonadisme décelable par les méthodes classiques de diagnostic- dans certaines situations cliniques et sous réserve d'une surveillance adaptée. Néanmoins le caractère très récent de ces acquisitions nécessite leur validation à travers des séries plus larges et suffisamment de recul pour asseoir l'efficacité et l'innocuité de l'androgénothérapie sous ses nouvelles formes et dans ses nouvelles indications.

\section{REFERENCES}

1. ANDERSON R., BANCROFT J., WU F. : The effects of exogenous testosterone one sexuality in normal men. J. Clin. Endocrinol. Metab. 1992, 75 : 1503-1507.

2. ARON C. : La Neurobiologie du Comportement Sexuel des Mammiferes. ed Hermann. Paris, 1984, VIII : $57-107$

3. BALS-PRATSCH M., KNUTH U.A., YOON Y.D., NIESCHLAG E. : Transdermal Testosterone substitution Therapy for Male Hypogonadism. The Lancet, 1986, $25: 943-946$.
4. BANCROFT J. : Endocrinology of sexual fonction. Clin. Obstet. Gynecol. 1980, 7 : 253-281

5. BANCROFT J. : Hormones and human sexual behavior. J. Sex. Marital Ther. 1984, 10 : 3-21.

6. BANCROFT J. : Sexual desire and the brain. Sex. Marit.Ther. 1988, $3: 11-27$.

7. BARDIN C.WAYNE., SWERDLOFF R.S., SANTEN R.J. : Androgens : Risks and Benefits. J. Clin. Endocrinol. Metab. 1991, 73, 1 : 4-7.

8. BEHRE H.M., NIECHLAG E.: Testosterone Buciclate (20 A et 1) in Hypogonadal Men : Pharmacokinetics and Pharmacodynamics of the New Longacting Androgen Ester. J. Clin. Endocrinol. Metab. $1992,75,5: 1204-1210$.

9. BHASIN S., SWERDLOFF RS., STEINER B, et al. : A biodegradable testosterone microcapsule formulation provides uniform eugonadal levels of testosterone for 10-11 weeks in hypogonadal men. J. Clin. Endocrinol. Metab. 1992, 74 : 75-83.

10. BONNET P., REITER E., BRUYNINX M. : Benign Prostatic Hyperplasia and Normal Prostate Aging : Differences in Types I and II $5 \alpha$-Reductase and Steroid Hormone Receptor Messenger Ribonucleic Acid (mRNA) Levels, but not in Insulin-Like Growth Factor mRNA Levels. J. Clin. Endocrinol. Metab. 1993, 77, 5 : 1203-1208.

11. BOUDON CH , TERRAZA A. , NAVARRO M., et al. : Activité $5 \alpha$-réductase des cellules de prostate humaine en culture. Andrologie, 1993, 3, $1: 50$.

12. BURRIS A.S., EWING L.L., SHERINS R.J. : Initial trial of slow-release testosterone microspheres in hypogonadal men. Fertil. steril. 1988, $50: 493-$ 497.

13. BUVAT J., BUVAT-HERBAUT M., LEMAIRE A., MARCOLIN G. : Hormone, Neurotransmetteurs Centraux et Comportement Sexuel de l'Homme. In : Arvis G, ed. Andrologie, tome III, Paris : Maloine, 1991 : 1661-1689

14. BUVAT J. : Neurotransmetteurs cérébraux et contrôle du comportement sexuel masculin. Andrologie, 1996, 6, 2 : 184-199.

15. CARANI C., BANCROFT J., GRANATA A., DEL RIO G., MARRAMA P. : Testosterone and Erectile Function, Nocturnal Penile Tumescence and Rigidity, and Erectile Reponse to Visual Erotic Stimuli in Hypogonadal and eugonadal Men. Psychoneuroendocrinology, 1992,17, $6: 647-654$.

16. CARRIE J.BAGATELL., HERMEN JR., RIVIER JE., BREMNER W.J. : Effects of Endogenous Testosterone and Estradiol on Sexual Behavior in Normal Young Men. J.clin.Endocrinol. Metab. 1994, 78, $3: 711-716$. 
17. CARRIE J.BAGATELL., HERMEN J.R., MATSUMOTO A.M., RIVIER J.E., BREMNER W.J. : Metabolic and behavioral effects of high-dose exogenous testosterone in healthy men. J. Clin. Endocrinol. Metab. 1994, 79, $2: 561-567$.

18. DEVIGNES D., MAHMOUD W., GUECHOT J., ARVIS G., GIBOUDEAU J. : Intérêt du dosage de la testostérone biodisponible dans les dysérections. Andrologie, 1996, 6 , 2 : 200-207.

19. GOOREN L.J.C. : Human male sexual functions do not require aromatisation of testosterone : a study using tamoxifen, testolactone and dihydrotestostérone: Arch.sex.behav, 1985, $14: 539-548$.

20 . GRIFFING G.T.: Editorial: Dinosaurs and steroids. J. Clin. Endocrinol. Metab., 1993, 77, 6 : 1450-1451.

21. HAFFNER S.M., MYKKANEN L., VALDEZ R.A., KATZ M.S. : Relationship of Sex Hormones to Lipids and Lipoproteins in Non diabetic Men. J. Clin. Endocrinol. Metab., 1993, 77, 6 : 1610-1615

22. HANDELSMAN D.J., CONWAY A.J., BOYLAN L.M. : Pharmacokinetics and pharmacodynamics of testosterone pellets in men. J. Clin. Endocrinol. Metab., 1990, $70: 216-221$.

23. HEATON J.P.W., SCHWAN J.VARRIN : Effects of Castration and Exogenous Testosterone Supplementation in an Animal Model of Penile Erection. J. Urol, 1994, $151: 797-800$.

24. HOLMANG A., SVEDBERG J., JENNISCHE E., BJORNTORP P. : The effects of testosterone on muscle insulin-sensitivity and morphology in female rats. Am. J. physiol, 1990, 259 : E 555- E 560.

25. HOLMANG A., BJORNTORP P. : The effects of testosterone on insulin sensitivity in male rats. Acta Physiol. Scand., 1992, 140 : 505-510.

26.HOOP R.C., PLYMATE S.R. : Regulation of sex hormone binding globulin gene expression by steroid and peptides hormones. Proc. of the 8th int. Congr of hormonal steroids. 1990.

27. KWAN M., GREENLEAF WJ., MANN J., CRAPO L., DAVIDSON J.M. : The nature of androgen action on male sexuality : combined laboratoryself-report study on hypogonadal men. J. Clin. Endocrinol. Metab., 1983, 57 : 557-562.

28. LEJEUNE H., DECHAUD H. : Dosage de la testostérone plasmatique chez l'homme. Andrologie, $1994,4,2: 216-222$.

29. LIU XIN-HUA, STEVEN WILEY H., WAYNE MEIKLE A. : Androgens Regulate Proliferation of Human Prostate Cancer Cells in Culture by Increasing Transforming Growth Factor-a (TGF- $\alpha$ ) and Epidermal Growth Factor (EGF)/TGF- $\alpha$ Receptor. J. Clin. Endocrinol. Metab., 1993, 77, 6 : 1472-1478.
30. LUISI M., FRANCHI R. : Double-blind group comparative study of testosterone undecanoate and mesterolone in hypogonadal male patients. J. endocrinol. invest., 1980, $3: 305-308$.

31. MATSUMOTO A.M. : "Andropause" Are reduced Androgen Levels in Aging Men Physiogically Important? WJM, 1993, 159, $5:$ 618-620.

32. MCCONNELL J., BARTSCH G., DEBRUYNE F., LE DUC A., MAHLER C. PAVONE-MACALUSO M. et al : Hormonal treatment of benign prostatic hyperplasia : Proc. of the international consultation on benign prostatic hyperplasia (BHP). Paris, 1991, 179-191.

33. McCONNELL J.D., WILSON J.D., GEORGE F.W., GELLER J., PAPPAS F., STONER E. : Finasteride, an Inhibitor of $5 \alpha$-Reductase, Suppresses Prostatic Dihydrotestosterone in Men with Benign Prostatic Hyperplasia. J.Clin. Endocrinol. Metab., 1992, 74, $3:$ 505-508.

34. MOSS H.B., PANZAK G.L., TARTER R.E. : Sexual Functioning of Male Anabolic Steroid Abusers. Arch Sex Behav., 1993, 22,1:1-12.

35. MOWSZOWICZ I., BERTHAUT I., MESTAYER CH., WRIGHT F., KUTTENN F, MAUVAIS-JARVIS P. : $5 \alpha$-Reductases: Physiologie et Pathologie. Andrologie 1994, $4,1:$ :11-77.

36. NIECHLAG E., BEHRE HM. : Comparative pharwiacokinetics of testosterone preparations : application of computer analysis and simulation. In : NIECHLAG E., BEHRE HM., eds. Testosteroneaction, deficiency, substitution, Berlin: Heidelberg, New york : Springer-Verlag: 115-35.

37. O'CARROLL R., BANCROFT J. : Testosterone therapy for low sexual interest and erectile dysfunction in men : A controlled study. 1984, Br. J. psychiat, $145: 146-151$.

38. PASQUALI R, CASIMIRRI F, CANTABELLI S., et al : Effects of obesity and body fat distribution on sex hormones and insulin in men: Metabolism, $40: 101-104$.

39. PHILLIPS G. : Relationships between serum sex hormones and the glucose-insulin-lipid defect in men with obesity. Metabolism. 1993, 42 : 116-120.

40.RAFEEQ A.S., BOUCHER A.E., MANNI A., SANTEN R.J., BARTHOLOMEW M., DEMERS L.M. : Transdermal Testosterone Therapy in the Treatment of Male Hypogonadism. J. Clin. endocrinol. Metab.,1988, 66, 3 : 546-551.

41. ROBEL P. : Recepteur et Mécanismes d'Action des Androgènes. Andrologie, 1993, 3, $4: 51-57$.

42. ROBEL P., GIULLIANO F., RAMPIN O. : Risques potentiels des inhibiteurs de la 5a-reductase pour le comportement sexuel masculin. Andrologie, $1995,5,2: 230-235$. 
43. SALMINIES S., KOCKOTT G., PIRKE KM., VOGT HJ., SCHILL WB. : Effects of testosterone remplacement on sexual behavior in hypogonadal men. Arch. Sex. Behav., 1982, 11 : 345-353.

44. SIMPKINS J.W., KALRA P.S., KALRA S.P. Variable effects of testosteron on dopamin activity in several microdissected regions in the preoptic ares and medial basal hypothalamus. Endocrinology, 1983, $112: 665$.

45. SMITH E.P., BOYD J., FRANK G.R. et al. : Estrogen resistance caused by a mutation in the estrogen-receptor gene in man. New Engl. J. Med., 1994, 331 : 1056-1061.

46. STEFANICK M.L., WILLIAMS P.T., KRAUSS R.M., TERRY R.B., VRANIZAN K.M., WOOD P.D. : Relationships of plasma estradiol, testostérone, and sex hormone-binding globuline with lipoproteine, apolipoproteine and high density lipoproteine subfractions in men. J. clin. Endocrinol. Metab., 1987, $64: 723-729$.

47. VITTO G.A., KAUFMAN J.M., VERMEULEN A. : Pathogenis of the decreased androgen levels in obese men. J. clin. Endocrinol. Metab., 1994, 79, $4: 997$.

\section{ABSTRACT}

Androgens and male sexuality

\section{A. Hafidi, M.M. Gharbi}

Testosteron is known to be critical for the right maintenance of masculin sexuality. It acts on all components of sexuality : libido, erection and ejaculation. As far as erection is concerned, one has to emphasize on the fact that only spontaneous nocturnal erections are androgen-dependent unlike erectile responses to visual erotic stimuli

The $T$ can act, at the level of target organes, either as unchanged or as more often observed after reduction to DHT or aromatisation to $\mathrm{E2}$. It is essencially as DHT that the male hormone seems to act on the sexuality at both the central and peripherical levels. The oestrogens even if now shown to have no peripherical action, are still subject to controversy as far as their central action is concerned. Therefore an action at the cerebral level of the oestrogens produced locally can not be definitively eliminated.

The concentrations of $T$ required for the re-establishment of the sexual function in case of hypogonadism are closer to the lower limits of normal values. The upper treshold of the action of $T$ on the sexuality, if any, would be at supra-physiological levels.

Androgenotherapy of sexual dysfonctionnalities is wittnissing a constant evolution. New and more performing products, as well as novel and less astreignant ways of administration, are already disponible or under evaluation. Moreover, the improvement of plasmic androgen exploration (dosage of free and non-sex hormone-binding globulin bound testosterone) associated to a better understanding of side effects of the androgenic therapeutics may allow in the near futur to reach a consensus on the androgenotherapy indications. As a matter of fact among the population of patients considered eugonadic (based on normal levels of T) and showing a sexual dysfonctionality, two subgroups might be interested : That of elderly patients displaying either an increase of the hormone-binding globulin (SHBG) or a decrease of the non-sex hormone-binding globulin bound testosterone levels and that of young patients suffering from idiopathic impotence. It should be, however, pointed at that the recent character of the different physiopathologic and therapeutic acquisitions requires a validation through larger series and long term studies.

Key words : Androgen, libido, erection, ejaculation, prostate, cardiovascular risk. 